\title{
Ensino de Ciências no contexto da Astronomia: o uso do Stellarium como objeto virtual de aprendizagem nos anos finais do Ensino Fundamental
}

\author{
Science teaching in the context of Astronomy: the use of Stellarium as a virtual learning object in \\ the final years of Elementary School
}

Enseñanza de las Ciencias en el contexto de la Astronomía: el uso de Stellarium como objeto de aprendizaje virtual en los últimos años de la Escuela Primaria

\author{
Nairys Costa de Freitas \\ ORCID: https://orcid.org/0000-0002-0799-8489 \\ Instituto Federal de Educação, Ciência e Tecnologia do Ceará, Brasil \\ E-mail: Nairys.freitas07@aluno.ifce.edu.br \\ Hugo Ferreira Alves \\ ORCID: https://orcid.org/0000-0002-4746-465X \\ Instituto Federal de Educação, Ciência e Tecnologia do Ceará, Brasil \\ E-mail: hugo.alves02@aluno.ifce.edu.br \\ José Ademir Damasceno Junior \\ ORCID: https://orcid.org/0000-0003-4110-532X \\ Secretaria de Educação do Estado do Ceará, Brasil \\ E-mail: profademir7@gmail.com \\ Mairton Cavalcante Romeu \\ ORCID: https://orcid.org/0000-0001-5204-9031 \\ Instituto Federal de Educação, Ciência e Tecnologia do Ceará, Brasil \\ E-mail: mairtoncavalcante@ifce.edu.br
}

\begin{abstract}
Resumo
O presente artigo tem como objetivo analisar as potencialidades do Objeto Virtual de Aprendizagem (OVA) Stellarium, nos anos finais do Ensino Fundamental, no que diz respeito ao ensino de conceitos básicos de Astronomia, na disciplina de Ciências, levando em consideração as limitações no Ensino de Ciências. O artigo se trata de uma revisão bibliográfica, a qual desenvolve uma reflexão sobre o que os pesquisadores reiteram a respeito das consequências que a falta de contextualização no ensino de Ciências vem causando na formação básica dos jovens, apresentando a popularização do ensino de Ciências, por meio da abordagem de conceitos básicos de Astronomia que possuem relação com a Base Nacional Comum Curricular (BNCC), como uma valiosa estratégia para superar esse obstáculo, entre outros. Além disso, relata os desafios do ensino de Ciências nos dias atuais, envolvendo a falta de recursos, erros conceituais nos livros didáticos e a formação acadêmica dos professores de Ciências. Sendo assim, a inclusão da Astronomia no Ensino de Ciências, envolve aspectos motivacionais e abrange várias áreas de conhecimento, além disso, possui popularidade significativa através do avanço da divulgação científica nos meios de comunicação. Diante das limitações de espaço e materiais didáticos, o Stellarium possui recursos que potencializam e possibilitam os tópicos de Astronomia na educação formal. Além disso, possibilita ao professor apresentar aos estudantes diversos fenômenos astronômicos, sendo este importante na ausência de telescópios, lunetas, binóculos ou mesmo na falta de espaço para a elaboração das atividades, despertando os estudantes para a alfabetização científica. Palavras-chave: Ensino de astronomia; Ensino de ciências; Enculturação científica; Objeto virtual de aprendizagem.
\end{abstract}

\begin{abstract}
This article aims to analyze the potential of the Virtual Learning Object (VLE) Stellarium, in the final years of elementary school, regarding the teaching of basic concepts of astronomy, in the discipline of science, taking into account the limitations in science teaching. The article is a literature review, which develops a reflection on what researchers reiterate about the consequences that the lack of contextualization in science teaching has caused in the basic education of young people, presenting the popularization of science teaching, through the approach of basic concepts of astronomy that are related to the Common National Curricular Base (BNCC), as a valuable strategy to overcome this obstacle, among others. In addition, it reports the challenges of science teaching today, involving the lack of resources, conceptual errors in textbooks, and the academic training of science teachers. Thus, the inclusion of astronomy in science teaching involves motivational aspects and covers various areas of knowledge, besides having significant popularity through the advancement of scientific dissemination in the media. Given the limitations of space and teaching materials, Stellarium has resources that enhance and enable Astronomy topics in formal education. In
\end{abstract}


addition, it allows the teacher to present to students several astronomical phenomena, which is important in the absence of telescopes, lunettes, binoculars, or even in the lack of space for the development of activities, awakening students to scientific literacy.

Keywords: Astronomy teaching; Science teaching; Scientific culturation; Virtual learning object.

\section{Resumen}

Este artículo pretende analizar las potencialidades del Objeto Virtual de Aprendizaje (OVA) Stellarium, en los últimos cursos de primaria, en relación con la enseñanza de conceptos básicos de astronomía, en la disciplina de ciencias, teniendo en cuenta las limitaciones en la enseñanza de las ciencias. El artículo es una revisión bibliográfica, que desarrolla una reflexión sobre lo que los investigadores reiteran acerca de las consecuencias que la falta de contextualización en la enseñanza de las ciencias viene provocando en la educación básica de los jóvenes, presentando la divulgación de la enseñanza de las ciencias, a través del abordaje de conceptos básicos de astronomía que se relacionan con la Base Curricular Nacional Común (BNCC), como una estrategia valiosa para superar este obstáculo, entre otros. Además, informa de los retos de la enseñanza de las ciencias en la actualidad, que implican la falta de recursos, los errores conceptuales de los libros de texto y la formación académica de los profesores de ciencias. Así, la inclusión de la astronomía en la enseñanza de las ciencias implica aspectos motivacionales y abarca diversas áreas de conocimiento, además, tiene una importante popularidad por el avance de la divulgación científica en los medios de comunicación. Dadas las limitaciones de espacio y material didáctico, Stellarium dispone de recursos que mejoran y posibilitan los temas de astronomía en la educación formal. Además, permite al profesor presentar a los alumnos diversos fenómenos astronómicos, siendo esto importante en ausencia de telescopios, lunetas, prismáticos o incluso en la falta de espacio para la elaboración de actividades, despertando a los alumnos a la alfabetización científica.

Palabras clave: Enseñanza de la astronomía; Enseñanza de las ciencias; Enculturación científica; Objeto virtual de aprendizaje.

\section{Introdução}

A presente pesquisa faz uma reflexão inicial a respeito do ensino de Ciências, evidenciando os desafios e limitações presentes nos anos finais do Ensino Fundamental. Fourez (2002) faz uma crítica ao ensino de Ciências sem contextualização na educação básica, ressaltando o desinteresse dos jovens em estudar áreas afins, falta de recursos didáticos, limitações na formação docente e erros conceituais nos livros didáticos. Além disso, para Carvalho (2007), as limitações no Ensino de Ciências impossibilitam o desenvolvimento da aprendizagem dos estudantes, não despertando a curiosidade, valorizando-se apenas a ação de decorar conceitos e fórmulas em vários níveis de ensino.

Diante das dificuldades no ensino de Ciências, Kator (2001), Langhi (2004) E Langhi (2009) apresentam os tópicos de Astronomia como uma excelente ferramenta na contextualização dos conteúdos de Ciências, evidenciando o seu potencial motivacional e interdisciplinar. No entanto, os referidos autores não apresentam propostas para que alguns obstáculos encontrados no ensino de conceitos básicos de Astronomia sejam superados ou mitigados. Infelizmente, tais limitações não são mencionadas nesses trabalhos, a fim de fomentar discussões sobre possíveis soluções balizadas na literatura da área, com vistas a popularizar e favorecer o ensino de conceitos básicos de Astronomia nas escolas brasileiras.

A Base Nacional Comum Curricular (Brasil, 2018) considera que a inserção das Tecnologias no ensino de Ciências possibilita o desenvolvimento de habilidades científicas e tecnológicas, aprofundando o conhecimento através da interdisciplinaridade, a fim de conhecer a dinâmica da natureza, dando importância o papel das novas tecnologias no ensino de Ciências. Sendo assim, o presente artigo traz a seguinte pergunta central: nos anos finais do Ensino Fundamental, como a ferramenta tecnológica "Stellarium” pode favorecer o ensino de Ciências?

Ao identificar nos argumentos dos pesquisadores citados neste trabalho em relação às dificuldades no ensino de Ciências, o artigo tem como objetivo analisar as potencialidades do Stellarium como ferramenta facilitadora do processo ensino-aprendizagem no ensino de Ciências, por meio da abordagem de conceitos básicos de Astronomia. 


\section{Uma Reflexão Sobre o Ensino de Ciências e a Enculturação Científica}

Diante de uma reflexão sobre a educação científica Fourez (2002), assegura que os jovens da atual geração questionam a importância da Ciência na cultura, sociedade e economia. O autor ainda questiona a respeito de nós, professores estarmos preparados para convencer os estudantes sobre a importância da Ciência. Em seu artigo sobre "a crise na ciência", Fourez (2002) destaca que, embora os jovens considerem a Ciência como uma realização humana, os alunos ainda não estão preparados para se dedicarem aos estudos de cunho científico. Carvalho (2007) defende que a dicotomia entre o que é Ciência e como ela está sendo ensinada desde os anos iniciais do Ensino Fundamental tem provocado várias reflexões entre pesquisadores da área, a respeito do processo de enculturação científica. Sendo assim, é importante observar que se torna difícil para os alunos aprenderem ciência que, na maioria das vezes, não está de acordo com o cotidiano e o senso comum (Brasil, 1998).

Contudo, Fourez (2002), em suas palavras, ratifica que as formações em licenciaturas estavam mais preocupadas em formar técnicos em Ciência do que fazê-los educadores. Carvalho (2007) assevera que a Ciência apresentada nas escolas não aborda a importância da diversidade cultural, conhecimentos históricos e sociais, os quais despertam a curiosidade, evitando uma tradição no ato de decorar em diversos níveis de ensino. Vale ressaltar que os conceitos decorados não são usados nem nas próprias classes dessas áreas. Sendo assim,

Ciência e Tecnologia são herança cultural, conhecimento e recriação da natureza. Ao lado da mitologia, das artes e da linguagem, a tecnologia é um traço fundamental das culturas. Por exemplo, conhece-se o período paleolítico pelo domínio do fogo e pelo uso da pedra lascada como instrumento de caça e pesca, substituído pela pedra polida no período neolítico, marcado pelo desenvolvimento da agricultura, da criação de animais e a utilização do ouro e do cobre. (Brasil, 1998, p. 23).

Nessa perspectiva, Bueno et al. (2020) analisa o espaço ocupado sobre os céus indígenas em documentos educacionais nacionais, apontando a importância do conhecimento produzido pelos povos indígenas e Astronomia cultural no Brasil. Contudo, os autores concluem que existe pouca representatividade da cultura indígena nos currículos. Os Parâmetros Curriculares Nacionais (PCN) de Ciências para o Ensino Fundamental (Brasil, 1998), orientam que a Ciência e a Tecnologia são herança cultural, corroborando o que Cazelli \& Franco (2001) estudaram a respeito do alfabetismo científico e a sociedade contemporânea, em que certificam que a educação não pertence apenas ao espaço escolar ou ao sistema de ensino, mas é reconhecida pelo seu potencial estruturador e impulsionador de toda a sociedade.

Para Carvalho (2007), quando existem aulas experimentais, são limitadas ao método científico, podendo ser planejadas para que os estudantes resolvam um problema experimental, procurando soluções que possibilitem a presença de alguns aspectos culturais e motivacionais no ensino.

Como solução para as limitações no Ensino de Ciências, vários pesquisadores consideram a Astronomia uma disciplina com alto grau motivacional, a qual estimula a autonomia dos estudantes e os aproxima da Ciência. Alguns desses trabalhos são: Kator (2001), Langhi (2004) E Laghi (2009). Martins E Recena (2021), ressaltam a importância da inclusão da Astronomia tanto no Ensino Fundamental quanto no Ensino Médio.

\section{A Astronomia nos Anos Finais do Ensino Fundamental}

A Astronomia é considerada a mais antiga das ciências, os primeiros registros astronômicos foram feitos pelos chineses, babilônios, assírios e egípcios, há cerca de 3.000 a.C. Os primeiros registros tratavam-se de estudos com objetivos práticos, como por exemplo: fazer calendários, otimizar o trabalho agrícola, eram bastante utilizados na astrologia, pois acreditavam que os deuses do céu tinham o poder da colheita, da chuva e até mesmo da vida (Oliveira Filho \& Saraiva, 2014). 
Por volta do século III a.C., na cidade egípcia de Alexandria, considerada uma grande metrópole daquele tempo, foi descoberto que a Terra é um mundo pequeno. Tais descobertas foram importantes para a humanidade, assim como as demais descobertas científicas. Além disso, a espécie humana é curiosa, valente e promissora, tendo feito descobertas inesperadas sobre o cosmos e o nosso lugar nele. Na visão do autor, as explorações do cosmos fazem lembrar o quanto a humanidade evoluiu, o qual defende que o conhecimento é um pré-requisito para a sobrevivência. (Sagan, 1980).

Levando em consideração a necessidade desse conhecimento, nos anos finais do Ensino Fundamental, são priorizadas as vivências, saberes, interesses e curiosidades dos alunos sobre o mundo em que estão inseridos. Contudo, ao longo desse processo, a ampliação progressiva e a capacidade de abstração da autonomia de ação e de pensamento vêm sendo perceptíveis, havendo aumento de interesse dos alunos pela vida social e pela busca de uma identidade própria. (Brasil, 2018). Logo,

Os alunos, no ensino Fundamental, ao estudaram o componente curricular de Ciências, aprenderão mais sobre eles próprios, acerca de outras espécies, quanto à manutenção da vida. Conhecer também sobre o nosso planeta, o Sistema Solar e o Universo possibilitarão que os mesmos tenham melhor propriedade para argumentar, defender e promover intervenções com o intuito de manter o equilíbrio na natureza (Damasceno Júnior \& Romeu, 2019, p. 9).

A Astronomia é reconhecida pelo seu alto grau de interdisciplinaridade, podendo ser aplicada em várias áreas de conhecimento (Matemática, Física, Química, Biologia, História, Geografia, Filosofia, entre outras), as quais podem proporcionar aos estudantes uma visão ampliada do conhecimento, podendo ser considerada uma disciplina integradora de conhecimentos. Com o avanço científico-tecnológico, a falta de ritmo entre este e o ensino das disciplinas da área de Ciências da Natureza, são perceptíveis. Dessa forma, os autores reconhecem a necessidade de enfocar os tópicos de Astronomia, relacionados às conquistas tecnológicas contemporâneas, pois o homem faz parte desse momento histórico e suas condições de vida dependem direta ou indiretamente deste conhecimento. (Dias \& Rita, 2008).

Além do seu caráter interdisciplinar, para Garcia, Prado e Nardi (2020) a Astronomia é um ramo das Ciências Naturais que possui um potencial motivador, por despertar a curiosidade dos estudantes diante de algumas temáticas bastante comuns nos canais de comunicação, as quais são: buracos negros, cosmologia e exploração do Sistema Solar. Segundo Langhi \& Martins (2018), a motivação traz ao indivíduo diversas opções, as quais possibilitam resultados satisfatórios em suas escolhas assertivas, uma vez que ela aumenta o nível de interesse e compreensão, gerando aprendizagem. Mesmo diante das potencialidades do ensino de Astronomia, observar o céu noturno é um desafio devido ao excesso de luminosidade.

As pessoas já não observam o céu noturno como antes porque estamos perdendo mais uma beleza natural: o céu estrelado, pois o excesso de luminosidade urbana provoca mais uma espécie de poluição, a poluição luminosa. Um céu noturno comum, sem luar e nuvens, poderia apresentar a visão de cerca de cinco mil estrelas, mas a iluminação dos postes públicos, monumentos e empresas as ofuscam de tal maneira que apenas mal se observa umas 200 estrelas, ou menos, dependendo das dimensões da cidade (Langhi \& Nardi, 2013. p. 110).

Sendo assim, é possível facilitar a aprendizagem dos estudantes por meio do auxílio de softwares nas aulas conceituais. De acordo com Garcia et al. (2020), a observação do céu é indispensável, pois existem vários fenômenos que possibilitam a observação a olho nu. No entanto, para os autores, a fim de superar as dificuldades com relação aos horários, visibilidade e espaços, o uso de softwares é uma excelente alternativa.

\section{Objetos Virtuais de Aprendizagem como Recursos Digitais Educacionais}

Os Objetos Virtuais de Aprendizagem surgiram com o objetivo de identificar conteúdos educacionais na web, para serem manuseados em diversos níveis de ensino e plataformas (Júnior, 2016). Portanto, os Objetos Virtuais de Aprendizagem (OVA) dispõem de características e componentes que buscam solucionar uma diversidade de problemas existentes nos dias 
atuais inerentes ao armazenamento e distribuição de informações por meios digitais (Longmire, 2001). Diante disso, as Leis de Diretrizes e Bases da Educação Nacional/LDBEN (Lei no 9.394/1996), no capítulo II, seção III, art. 32, inciso II, afirma que a inserção das tecnologias na educação básica é uma iniciativa substancial para o Ensino Fundamental, a qual é atribuída à formação básica do cidadão por meio do entendimento para o avanço do ensino e da aprendizagem nas escolas. A definição de tecnologia no dizer de Saéz (1999, p. 15): "É o conjunto de conhecimentos relatos e cosmovisões que pressupõe qualquer aplicação técnica presente em diferentes contextos históricos, sociais e econômicos”. Entretanto,

Além do acesso e possibilidades variadas de leituras, o aprendiz que interage com o conteúdo digital poderá também se comunicar com outros sujeitos de forma síncrona e assíncrona em modalidades variadas de interatividade: um - um e um - todos comuns das mediações estruturados, vídeo, rádio e TV; e principalmente todos - todos, própria do ciberespaço (Santos, 2003, p. 4).

Como caracteriza Giordan (2005), as aplicabilidades da internet nas escolas nos dias atuais são consideravelmente diversificadas. Deveras, "Se a escola não inclui a Internet na educação das novas gerações, ela está na contramão da história, alheia ao espírito do tempo e, de forma delituosa, produzindo exclusão social ou exclusão da cibercultura" (Silva, 2005, p. 63). Outrossim, a aplicação dos recursos e ferramentas disponíveis nas plataformas virtuais proporcionam a administração dos conteúdos disponibilizados e dos processos de ensino e aprendizagem (Tori, 2010). Conforme Belloni (2001) afirma em seu trabalho, o professor tem como papel estimular e orientar os discentes na busca de novos conhecimentos, gerenciando as dificuldades inerentes ao uso das tecnologias e ao excesso de informações disponibilizadas.

\section{O Uso do Stellarium para Facilitar o Processo de Ensino e Aprendizagem}

$\mathrm{Na}$ educação formal, as abordagens relacionadas à Astronomia têm provocado alguns erros conceituais por parte de alunos e professores. Tais erros se dão devido à má qualidade dos livros didáticos, uso de recursos limitados e projeção de imagens planificadas, as quais evidenciam dúvidas a respeito dos fenômenos astronômicos (Garcia, Prado \& Nardi, 2020). Contudo,

[...] se a órbita elíptica da Terra fosse de fato tão excêntrica conforme as concepções alternativas de muitos alunos, professores e livros didáticos, o Sol deveria alterar perceptivelmente seu tamanho aparente no céu à medida que a Terra se aproximasse ou se afastasse dele (Langhi \& Nardi, 2007. p. 96).

Stellarium $^{1}$ é um planetário de código aberto o qual possibilita a visualização do céu em três dimensões, onde o observador terá uma visão semelhante a observação a olho nu, binóculo ou telescópio. Além disso, oferece os seguintes recursos que possibilitam visualizar o céu nos moldes de um planetário:

- catálogo padrão com mais de 600.000 estrelas;

- catálogos adicionais com mais de 177 milhões de estrelas;

- catálogo padrão com mais de 80.000 objetos de céu profundo;

- catálogo extra com mais de 1 milhão de objetos de céu profundo;

- $\quad$ asterismos e ilustrações das constelações;

- $\quad$ constelações de mais de 20 diferentes culturas;

- imagens de nebulosas (catálogo Messier $^{2}$ completo);

- via láctea realista;

- atmosfera, nascer e pôr-do-sol bastante realistas;

- os planetas e seus satélites.

\footnotetext{
${ }^{1}$ Planetário virtual, disponível em: <https://stellarium.org/pt/>. Acesso em 20 de maio de 2021.

${ }^{2}$ Catálogo astronômico compilado pelo astrônomo francês Charles Messier composto por aproximadamente 100 objetos do céu profundo, os quais são considerados os mais belos, incluindo nebulosas, aglomerados estelares e galáxias. Fonte: < https://planetario.ufsc.br/o-catalogo-messier/>.
} 
Genuino (2014) apresenta o Software Stellarium como uma excelente ferramenta, a qual possibilita simular fenômenos astronômicos, com o contexto histórico da Astronomia e a contagem do tempo através das observações dos corpos celestes. Longhini e Mora (2010) conceituam o Stellarium como Objeto Virtual de Aprendizagem (OVA), o qual auxilia no ensino de Astronomia, rompendo as limitações de espaço, materiais, acessibilidade, entre outros. Embora não seja voltado para a educação formal, o observatório virtual oferece recursos que facilitam o processo de ensino-aprendizagem, levando em consideração as limitações no ensino de Ciências Naturais (Genuino, 2014).

[...] à medida que se aproxima a conclusão do Ensino Fundamental, os alunos são capazes de estabelecer relações ainda mais profundas entre a ciência, a natureza, a tecnologia e a sociedade, o que significa lançar mão do conhecimento científico e tecnológico para compreender os fenômenos e conhecer o mundo, o ambiente, a dinâmica da natureza (Brasil, 2018, p. 343).

Além disso, a compreensão dos fenômenos da natureza através das tecnologias possui característica interdisciplinar, pois abrange habilidades específicas e tecnológicas (Brasil, 1998). Um dos conteúdos centrais selecionados nas Orientações Curriculares Nacionais - PCN (1998) para o Ensino Fundamental, a fim de desenvolver conceitos, procedimentos e atitudes, trata-se da valorização de conhecimentos históricos, levando em consideração o papel de novas tecnologias e ideias relacionadas aos principais eventos da história da Astronomia até os dias atuais.

Um dos recursos que o simulador possui é a ilustração de constelações de mais de 20 culturas, o qual é possível relatar que os astrônomos da antiguidade observavam os agrupamentos aparentes de estrelas ligadas por uma linha imaginária, as quais são chamadas de constelações com formato de figuras de pessoas, animais, ou objetos relacionados à cultura. Segundo Oliveira Filho e Saraiva (2014), o conceito de constelação surgiu durante a Pré-História, sendo usada para identificar as estações do ano e são representadas de acordo com as suas crenças ou mitologias durante as navegações, e por essa razão várias civilizações adotaram as suas próprias constelações.

Embora identificar as constelações não seja uma tarefa tão fácil numa observação real, elas nos ajudam a separar o céu em proporções menores (Oliveira Filho \& Saraiva, 2014). Genuino (2014) reitera que o Stellarium permite simular o céu em condições próximas à realidade, possibilitando informações sobre os corpos celestes e a visualização dos fenômenos astronômicos.

Figura 1: Visualização de algumas constelações através do Stellarium.

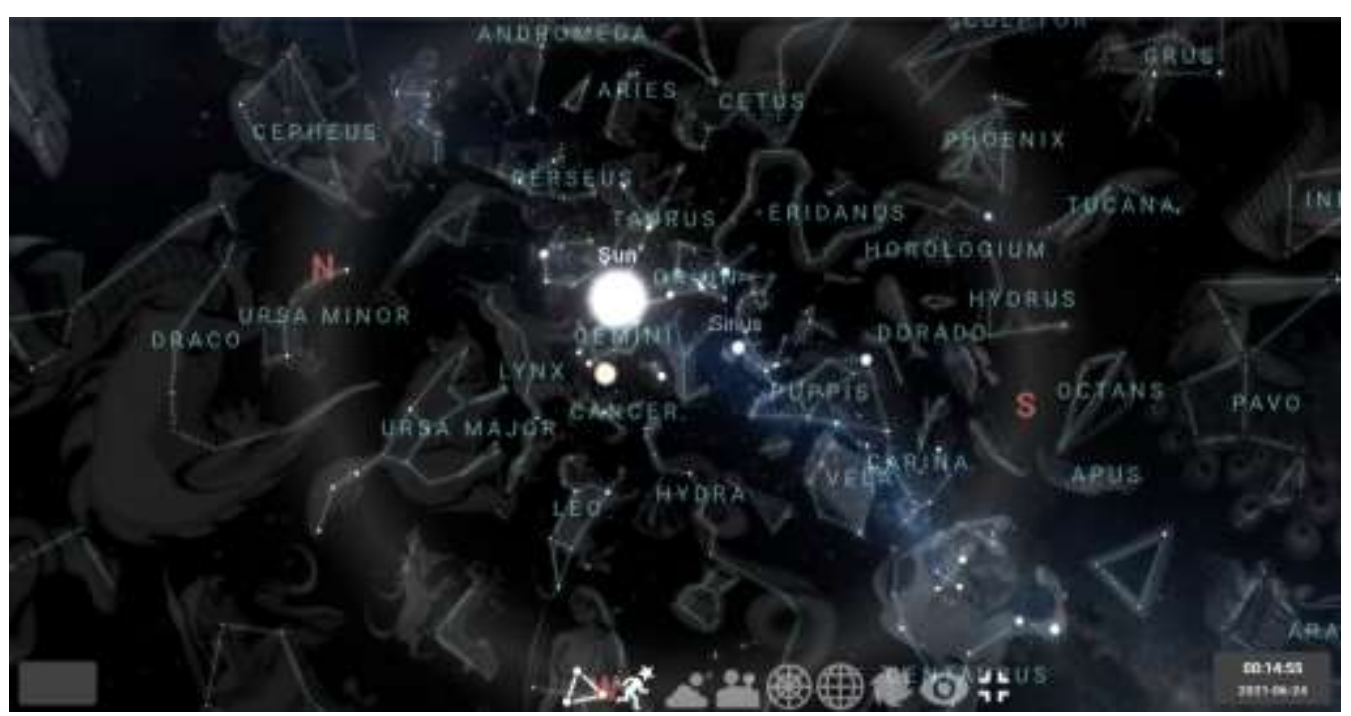

Fonte: <https://stellarium.org/pt/>. 
Na unidade temática Terra e Universo, são abordadas as características dos corpos celestes, suas dimensões, composição, localizações, movimentos e as forças atuantes. Além disso, tem como objetivo aprofundar as experiências sobre observação do céu, do planeta Terra, das zonas habitadas pelos seres vivos e fenômenos astronômicos (Brasil, 2018).

O Stellarium permite que sejam visualizados as estrelas, constelações, planetas, nebulosas, Sol e Lua em suas reais posições, exibindo o céu em data e hora especificadas, em diferentes graus de aproximação e ângulos. Possuindo os seguintes recursos em sua interface:

- Um zoom poderoso;

- $\quad$ Controle de tempo;

- Interface em diversos idiomas;

- $\quad$ Projeção olho-de-peixe ${ }^{3}$ para redomas de planetários;

- $\quad$ Projeção esférica-espelhada para sua própria redoma de baixo custo;

- $\quad$ Nova interface gráfica e controles de teclado extensíveis;

- Controle de telescópios.

Figura 2: Visualização da Lua através do Stellarium.

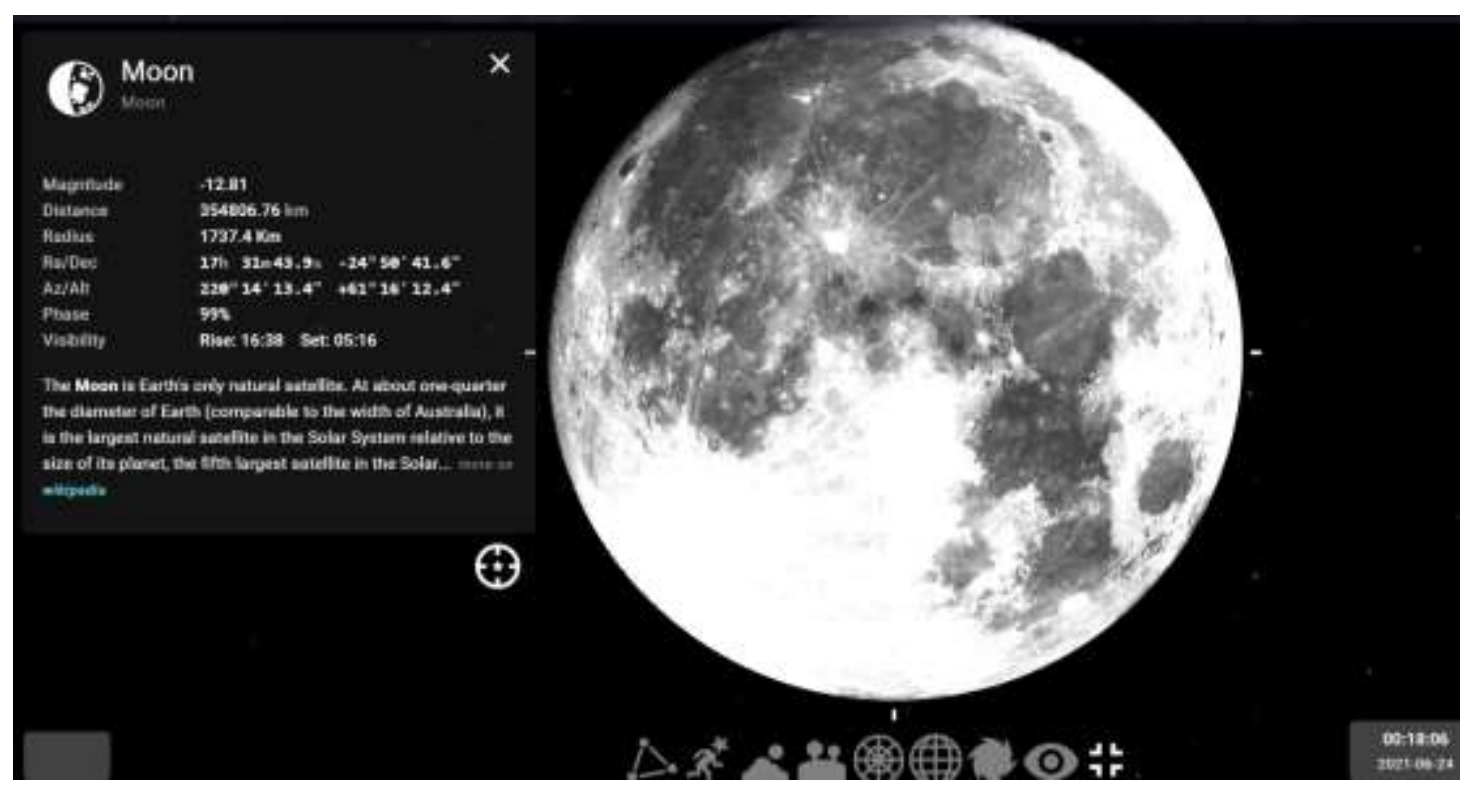

Fonte: $<$ https://stellarium.org/pt/>.

\footnotetext{
${ }^{3}$ Lente olho-de-peixe trata-se de uma grande angular, em que as imagens são esféricas e distorcidas, proporcionando maior profundidade de campo. Fonte: <https://fotografiaprofissional.org/lente-olho-de-peixe-fish-eye/>.
} 
Figura 3: Visualização das Plêiades através do Stellarium.

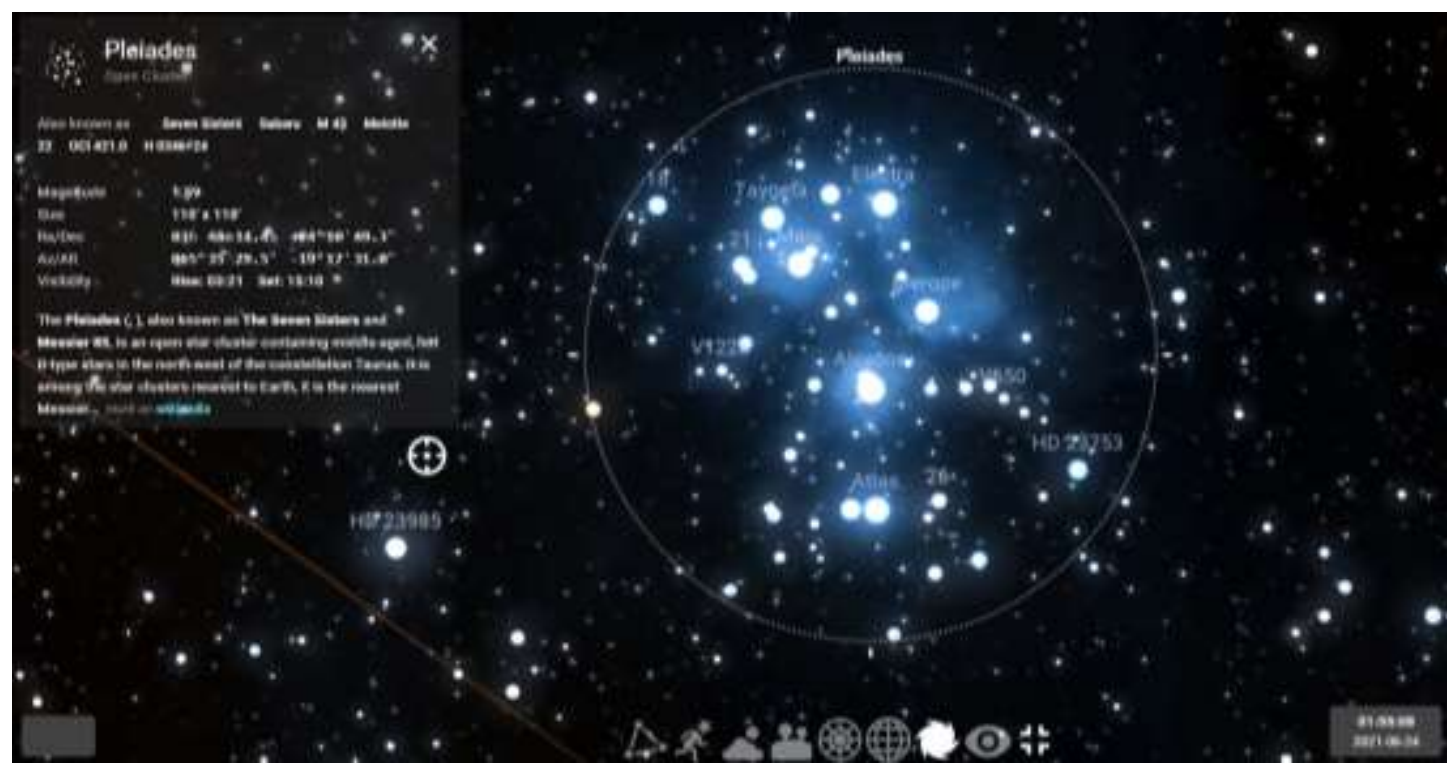

Fonte: <https://stellarium.org/pt/>.

Como descrito por Genuino (2014), o Stellarium é de fácil manuseio, o qual oferece compreensão na visualização dos corpos celestes, embora sendo difícil de interpretar alguns fenômenos, o planetário virtual possui recursos que proporcionam clareza na interpretação, apresentando as características dos astros em alta definição.

\section{Procedimentos metodológicos}

O presente trabalho possui caráter descritivo, tendo a pesquisa bibliográfica como estratégia (Boente \& Braga, 2004, Thomas, et al., 2012). A pesquisa bibliográfica é a base da investigação científica, em que o pesquisador faz buscas de obras já publicadas a fim de estudar o tema a ser investigado (Andrade, 2010). De acordo com os trabalhos Gil (2002), Lakatos e Marconi (2003), a pesquisa bibliográfica é caracterizada pelas fontes genuínas e concretas que respaldam a investigação a ser realizada.

A organização do trabalho se deu pela delimitação do tema, o qual motivou o levantamento de hipóteses através da leitura de artigos, trabalhos de conclusão de curso, livros, teses e dissertações, objetivando estudar a ferramenta tecnológica Stellarium como recurso didático para a inserção da Astronomia no ensino de Ciências nos anos finais do Ensino Fundamental. O levantamento das fontes bibliográficas teve como base os trabalhos (artigos, dissertações e teses), as quais foram identificadas através de pesquisas no google academic, em catálogos, editoras, revistas, teses e artigos, periódicos da CAPES, plataforma Sucupira e documentos oficiais que norteiam a elaboração do currículo do Ensino Fundamental.

\section{Considerações Finais}

A partir da pesquisa bibliográfica foi possível fundamentar a importância do uso de ferramentas tecnológicas, tencionando superar as limitações no ensino de Ciências, levando em consideração a preocupação dos pesquisadores com as possíveis consequências causadas pela falta de contextualização e democratização do conhecimento.

Contudo, nesta pesquisa, evidenciou-se que a abordagem de conceitos básicos de Astronomia favorece a contextualização dos conteúdos científicos, reforçando o seu potencial motivador e interdisciplinar. Ademais, por meio dessa estratégia, o professor será capaz de promover a alfabetização e enculturação científica na educação formal, consolidando a inserção da Astronomia na educação básica. 
Diante disso, considerando que muitas escolas não possuem recursos e espaço para a realização das aulas de Astronomia, havendo possibilidade de poluição luminosa em algumas cidades, o uso de Objetos Virtuais de Aprendizagem (OVA) é considerado uma excelente alternativa no processo ensino e aprendizagem. Sendo assim, foi realizado um estudo sobre as potencialidades do observatório virtual Stellarium na educação formal, em que foram constatados trabalhos que versam sobre o tema.

O Stellarium permite que os estudantes relacionem as diferentes leituras do céu com as culturas (caça, agricultura, mito, orientação espacial e temporal), além disso, permite ao professor usar os recursos para explicar a origem dos astros pertencentes ao Sistema Solar, constelações, aglomerados de estrelas e galáxias. Apesar de não ser uma ferramenta voltada para o ensino na educação formal, o Stellarium potencializa e possibilita a inserção dos tópicos de Astronomia no ensino de Ciências dos anos finais do Ensino Fundamental, sendo considerada uma ferramenta capaz de facilitar a compreensão dos alunos acerca dos conceitos científicos estudados na escola.

\section{Referências}

Andrade, M. M. (2010). Introdução à metodologia do trabalho científico: elaboração de trabalhos na graduação. Ed. Atlas.

Belloni, M. L. (2001). Educação a Distância. Ed. Autores Associados.

Boente, A., \& Braga, G. (2004). Metodologia científica contemporânea para universitários e pesquisadores. Brasport, $79-98$.

Brasil. (1996). Ministério da Educação (MEC). Lei de Diretrizes e Bases da Educação Nacional. Lei número 9394. Brasília DF.

Brasil. Ministério da Educação (MEC). (2018). Base Nacional Comum Curricular. Brasília DF.

Brasil. Secretaria de Educação Fundamental. (1998). Parâmetros curriculares nacionais: Ciências Naturais / Secretaria de Educação Fundamental. Brasília DF.

Bueno, M. A., Oliveira, E. A. G., Rodrigues, M. de S., \& Nogueira, E. M. L. (2020). Saberes indígenas sobre os céus: astronomia cultural em documentos educacionais brasileiros. Tear: Revista De Educação, Ciência E Tecnologia, 9(2).

Carvalho, A. M. P. (2007). Habilidades de professores para promover a enculturação científica. Revista Contexto e Educação, 22 (77), $25-49$.

Cazelli, S.; Franco, C. (2001). Alfabetismo científico: novos desafios no contexto da globalização. Ensaio - Revista em Educação em Ciências, 1(1), 1-16.

Damasceno Júnior, J. A. \& Romeu, M. C. (2019). O planetário como recurso didático para o ensino de astronomia e de uma alfabetização científica à luz da Base Nacional Comum Curricular. Tear: Revista de Educação, Ciência e Tecnologia, Canoas, 8 (1).

Dias, C. A. C. M., \& Santa Rita, J. R. (2008). Inserção Da Astronomia Como Disciplina Curricular Do Ensino Médio. Revista Latino-Americana De Educação Em Astronomia, (6), 55-65.

Fourez, G. (2002). Crise no ensino de Ciências? Investigação em ensino de ciências. 8 (2), 1-14.

Garcia, A. L. C, Prado, A. F \& Nardi, R. (2020). O software stellarium e o ensino de ciências astronômicas nos anos iniciais do ensino fundamental. $V$ Congresso Brasileiro de Ensino e Processos Formativos. Bauru (SP).

Genuino, L. C. C. (2014). O uso de tecnologias no ensino de Astronomia na educação básica. Monografia (Curso de Especialização Fundamentos da Educação: Práticas Pedagógicas Interdisciplinares da Universidade Estadual da Paraíba) - Universidade Estadual da Paraíba, Campina Grande, PB.

Gil, A. C. (2002). Como elaborar projetos de pesquisa. Ed. Atlas.

Júnior, A. W. (2016). Objetos virtuais de aprendizagem como recursos digitais educacionais. Pedagogia em Foco. Iturama, 11 (5), $53-65$.

Kantor, C. A. (2001). A ciência do céu: uma proposta para o ensino médio. Dissertação de Mestrado - Departamento de Física Experimental, Instituto de Física, Universidade de São Paulo, São Paulo.

Lakatos, E. M. \& Marconi, M. A. (2003). Fundamentos de Metodologia Científica. Ed. Atlas.

Langhi, R. \& Nardi, R. (2013). Educação em Astronomia: repensando a formação de professores. Escrituras Editora.

Langhi, R. \& Nardi, R. (2007). O ensino de Astronomia: erros conceituais mais comuns presentes em livros didáticos de ciências. Caderno Brasileiro de Ensino de Física. 24 (1).

Langhi, R. \& Martins, B. A. (2018). Um estudo exploratório sobre os aspectos motivacionais de uma atividade não escolar para o ensino da Astronomia. Caderno Brasileiro de Ensino de Física, 35 (1), 64-80. 
Research, Society and Development, v. 10, n. 15, e558101521999, 2021

(CC BY 4.0) | ISSN 2525-3409 | DOI: http://dx.doi.org/10.33448/rsd-v10i15.21999

Langhi, R. (2004). Um estudo exploratório para a inserção da Astronomia na formação de professores dos anos iniciais do Ensino Fundamental. Dissertação (Mestrado em Educação para a Ciência) - Faculdade de Ciências, UNESP, Bauru.

Langhi, R. (2009). Astronomia nos anos iniciais do ensino fundamental: repensando a formação de professores. Tese (Doutorado em Educação para a Ciência) - Faculdade de Ciências, UNESP, Bauru.

Longmire, W. (2001). A primer on learning objects. American Society for Training \& Development: Virginia, USA.

Longhini, M. D. \& Mora, I. M. (2010). Uma investigação sobre o conhecimento de Astronomia de professores em serviço e em formação. In: Longhini, M. D. (Org). Educação em Astronomia: Experiências e contribuições para a prática pedagógica. Campinas: Átomo, 87-116.

Martins, B. A. \& Recena, M. C. P. (2021). Análise dos aspectos motivacionais de estudantes em uma disciplina eletiva de Astronomia. Caderno Brasileiro de Ensino de Física, 38 (1), 16-44.

Saéz, V.M.M. (1999). Globalización, Nuevas Tecnologias y Comunicación. Ediciones de La Torre.

Santos, E. O. (2003). Ambientes virtuais de aprendizagem: por autorias livre, plurais e gratuitas. In: Revista FAEBA, 12 (18).

S. O. Kepler \& Saraiva. M. F. O. (2014). Astronomia \& Astrofísica. Porto Alegre: Departamento de Astronomia - Instituto de Física Universidade Federal do Rio Grande do Sul, 716 p.

Silva, M. Internet na escola e inclusão. In: Almeida, M. E. \& Moran, J. M. (2005). Integração das Tecnologias na Educação. Secretaria de Educação a Distância. Brasília: Ministério da Educação, Seed, 62-9.

Sagan, C. (1980). Cosmos. Ed. Francisco Alves.

Tori, R. (2010). Educação sem Distância: as tecnologias interativas na redução de distâncias em ensino e aprendizagem. Ed. Senac. 